\title{
Malaria is an uncommon cause of adult sepsis in south-western Uganda
}

Mary A Auma ${ }^{1}$, Mark J Siedner ${ }^{2}$, Dan Nyehangane ${ }^{3}$, Aisha Nalusaji ${ }^{3}$, Martha Nakaye ${ }^{4}$, Juliet Mwanga-Amumpaire ${ }^{1,3}$, Rose Muhindo ${ }^{1}$, L Anthony Wilson ${ }^{1}$, Yap Boum $\|^{1,3}$ and Christopher C Moore ${ }^{5^{*}}$

\begin{abstract}
Background: Malaria is often considered a cause of adult sepsis in malaria endemic areas. However, diagnostic limitations can make distinction between malaria and other infections challenging. Therefore, the objective of this study was to determine the relative contribution of malaria to adult sepsis in south-western Uganda.
\end{abstract}

Methods: Adult patients with sepsis were enrolled at the Mbarara Regional Referral Hospital between February and May 2012. Sepsis was defined as infection plus $\geq 2$ of the following: axillary temperature $>37.5^{\circ} \mathrm{C}$ or $<35.5^{\circ} \mathrm{C}$, heart rate $>90$ or respiratory rate $>20$. Severe sepsis was defined as sepsis plus organ dysfunction (blood lactate $>4 \mathrm{mmol} / \mathrm{L}$, confusion, or a systolic blood pressure $<90 \mathrm{mmHg}$ ). Sociodemographic, clinical and laboratory data, including malaria PCR and rapid diagnostic tests, as well as acid fast bacteria sputum smears and blood cultures were collected. Patients were followed until in-patient death or discharge. The primary outcome of interest was the cause of sepsis. Multivariable logistic regression was performed to assess predictors of mortality.

Results: Enrollment included 216 participants who were 51\% female with a median age of 32 years (IQR 27-43 years). Of these, 122 (56\%) subjects were HIV-seropositive of whom 75 (66\%) had a CD4+ T cell count $<100$ cells/ $\mu \mathrm{L}$. The prevalence of malaria was $4 \%$ (six with Plasmodium falciparum, two with Plasmodium vivax). Bacteraemia was identified in $41(19 \%)$ patients. In-hospital mortality was 19\% $(n=42)$. In multivariable regression analysis, Glasgow Coma Score $<9$ (IRR 4.81, 95\% Cl 1.80-12.8) and severe sepsis (IRR, 2.07, 95\% Cl 1.03-4.14), but no specific diagnoses were statistically associated with in-hospital mortality.

Conclusion: Malaria was an uncommon cause of adult sepsis in a regional referral hospital in south-western Uganda. In this setting, a thorough evaluation for alternate causes of disease in patients presenting with sepsis is recommended.

Keywords: Sepsis, Uganda, Africa, Malaria, Adult

\section{Background}

Severe sepsis is an important cause of death in subSaharan Africa (SSA) where the global burden of lethal infectious diseases is highest [1]. Due to resource limitations, including lack of investigative laboratory capacity, the aetiology of infections causing sepsis in this region is frequently unknown leading to empirical antimicrobial therapy. In malaria-endemic regions this frequently includes anti-malarial drugs. Although the majority of malaria rests with children and pregnant women, prevalence can also be high among non-pregnant adult populations,

\footnotetext{
* Correspondence: ccm5u@virginia.edu

${ }^{5}$ Department of Medicine, Division of Infectious Diseases and International Health, University of Virginia, Charlottesville, VA, USA

Full list of author information is available at the end of the article
}

particularly in those infected by HIV [2-6]. One recent report suggested that the prevalence of malaria in adults is higher than previously thought [7]. However, incidental parasitaemia can occur in asymptomatic patients so the contribution of parasitaemia to the course of severe sepsis can be difficult to discern. Furthermore, malaria is frequently over-diagnosed in areas such as Uganda with limited laboratory infrastructure. This can lead to overtreatment and development of resistance to antiparasitic medications $[8,9]$.

The time to the initiation of appropriate antimicrobial therapy is an important determinant of the outcome from severe sepsis [10]. On the other hand, indiscriminate use of antimicrobial therapy leads inexorably to antimicrobial resistance. Therefore, it is critical to determine 
the microbiological cause of severe sepsis and to focus antimicrobial therapy according to microbiological test results. In malaria-endemic settings, a better understanding of the contribution of malaria to severe sepsis would allow tailored empirical antimicrobial therapy and avoid overor under-treatment of malaria. Therefore, the objective of this study was to determine the contribution of malaria to sepsis in adults admitted to a regional referral hospital in south-western Uganda. Because rapid diagnostic tests (RDTs) have not been studied in adults with sepsis, a secondary aim was to determine the sensitivity and specificity of bedside malaria tests compared to a polymerase chain reaction (PCR) confirmed malaria diagnosis in the setting of adult sepsis.

\section{Methods}

\section{Site description}

The study was conducted on the medical ward of Mbarara Regional Referral Hospital (MRRH), which is the teaching hospital for the Faculty of Medicine at the Mbarara University of Science and Technology (MUST) and serves a largely rural population of 1.2-2.5 million people from surrounding districts in south-western Uganda. Mbarara District has an average malaria prevalence of $4 \%$ in urban areas and $23 \%$ in rural areas. The climate is tropical with a bimodal rainfall pattern in September-January and March-May, and an average precipitation of 1,200 mm per annum [11].

\section{Study population and definitions}

Adult patients (age $\geq 18$ years) admitted to the medical ward during the study period from February-May 2012 were screened consecutively during the hours of 08.00 0.00 . Patients were included if they met criteria for sepsis which was defined by clinical suspicion of infection and $\geq 2$ of the following: axillary temperature $>37.5^{\circ} \mathrm{C}$ or $<35.5^{\circ} \mathrm{C}$, heart rate $>90$ beats $/ \mathrm{min}$, and respiratory rate $>20$ breaths $/ \mathrm{min}$. White blood cell concentration (WBC) was not included in the enrolment criteria due to lack of immediate availability of results. Severe sepsis was diagnosed in patients with evidence of end-organ dysfunction (blood lactate $>4 \mathrm{mmol} / \mathrm{L}$, confusion, or a systolic blood pressure $<90 \mathrm{mmHg}$ ) [5]. Patients were excluded if they required triage to a surgical or obstetrics and gynaecology ward. Patients or their attendant (an accompanying family member or friend) provided informed consent prior to their enrolment in the study. All clinical and laboratory data were provided to the attending medical team as soon as they were available.

\section{Data collection}

A structured questionnaire was administered to patients or their attendants to collect the following data for each participant: demographics, history of presenting symptoms, and HIV serology status. Examination findings, including vital signs and Glasgow Coma Scale (GCS), and in-hospital mortality were also recorded. The study team followed each patient until discharge or death, but medical management was provided by the admitting medical team.

Laboratory investigations were obtained for each patient at the time of enrolment including complete blood cell concentration (Beckman Coulter, France), random blood glucose (Accu-Chek portable glucose analyzer; Roche diagnostics, Germany), and whole blood lactate (Accutrend Portable Lactate Analyzer; Sports Resource Group, USA). Prior to antibiotic administration, a blood sample was obtained aseptically and $10 \mathrm{~mL}$ was inoculated into brain heart infusion broth in duplicates and incubated at $37^{\circ} \mathrm{C}$ at the MUST Microbiology Laboratory. This laboratory is enrolled in the International Organisation for Standardisation and maintained satisfactory performance throughout the study period. Positive blood cultures were observed for turbidity, a clot or haemolysis; Gram's stain was done after subculturing on blood agar, MacConkey, and Chocolate agar plates for maximum recovery and morphological identification of the organisms. The plates were incubated at $37^{\circ} \mathrm{C}$ for $18-24 \mathrm{~h}$ aerobically and anaerobically for Chocolate agar. Blood cultures that did not show turbidity were further incubated up to 10 days. Biochemical identification of culture isolates was done according to standard methods. Bacteraemia was defined as bacterial isolation from one or more blood culture bottles. Cultures growing coagulase-negative staphylococci were considered contaminated and not included in the final analyses.

Patients with suspected tuberculosis had sputum obtained for light-emitting diode fluorescent microscopy (LED-FM). For patients whose HIV status was not known or documented at the time of admission, HIV counselling and testing was provided according to national guidelines. Accordingly, a rapid HIV test was done (Determine, Abbott Laboratories, Tokyo, Japan) followed by a confirmatory test for initially positive results (Statpak, Chembio Diagnostic Systems, Inc, Medford, USA). When the results were conflicting, a tie-breaker (Unigold, Trinity Biotech plc, Bray, Ireland) was used.

Malaria blood slides were evaluated via Field's staining as previously described [12]. For the purposes of quality control, $10 \%$ of all slides and all positive slides were double-read by a second experienced and blinded technician. Slides were also double-read in the case of a positive RDT result. In cases of discordant results between two microscopists or between RDT result and microscopy an additional reading was performed by a third microscopist and taken as the definitive microscopy result. A drop of blood was obtained by lancet for malaria RDT according to manufacturer's directions (SD Bioline Malaria Ag Pf/Pan, Standard Diagnostics, Inc, Korea). 
This RDT kit uses histidine-rich protein II (HRP-II) for identification of Plasmodium falciparum and Plasmodium lactate dehydrogenase (pLDH) for identification of Plasmodium vivax, Plasmodium ovale, and Plasmodium malariae.

Blood was also obtained by phlebotomy for PCR testing. Venous blood (100-200 $\mu \mathrm{L})$ was applied to Whatman FTA Classic Filter paper cards (GE Healthcare Ltd, New Jersey, USA) and left to air dry. These cards deactivate viral DNA/RNA and preserve human and parasite DNA for downstream analyses. DNA extractions were carried out according to the manufacturer's instructions. Briefly, a disc of $1.2 \mathrm{~mm}$ in diameter was punched from the centre of each dried blood spotted card and washed three times with Whatman FTA Purification Reagent, and twice with TE buffer. This treated disc was then used directly in subsequent PCR analyses. Nested PCR was performed using the primers and amplification conditions as previously described [13]. Nested PCRs were performed initially to screen for samples containing Plasmodium as described. Species of Plasmodium in positive samples were determined in separate species-specific nest 2 reactions. The resulting PCR products were visualized on $2 \%$ agarose gels, with the presence or absence of a band with each species primer pair indicative of the presence or absence of that species in the initial sample. The laboratory technicians performing PCR were blinded to the results of blood smears and RDTs.

\section{Statistical analysis}

Demographic, clinical and laboratory characteristics of the cohort were summarized. For the primary outcome of interest, the relative contribution of etiologies of sepsis was measured. The causes of sepsis were defined as either definitive or presumptive. Definitive causes were tuberculosis identified by sputum LED-FM, culture-proven bacteraemia, and malaria, as determined by a positive PCR. Presumptive causes of sepsis included malaria (peripheral blood smear or RDT), tuberculosis, community acquired pneumonia, enteric fever, fever of unknown origin and other or unspecified diagnosis. $\chi^{2}$ testing was used to compare baseline characteristics between those with and without a definitive diagnosis of malaria. Poisson regression models were fitted to determine factors associated with incident hospital mortality, including demographic characteristics, presenting signs, laboratory results, and discharge diagnoses. Finally, multivariable Poisson regression models which included all the characteristics that were significant in univariable models as determined by a p-value $<0.25$ were performed.

\section{Ethics statement}

Approval was obtained from the institutional review boards of MUST and the University of Virginia prior to the beginning of enrolment in this study.

\section{Results}

\section{Cohort characteristics}

A total of 646 patients were screened and 216 were enrolled (Figure 1). Their median age was 32 years (range 27-42 years) and approximately half were women (Table 1 ). HIV-infection was present in 56\% (122 of 216) of the cohort and $36 \%$ (44 of 122) were receiving antiretroviral therapy (Table 2). Of those patients who had a CD4+ $\mathrm{T}$ cell test performed, 66\% (75 of 113) had a CD4+ T-cell concentration $<100$ cells $/ \mu \mathrm{L}$. The majority of patients (90\%, 194 of 216) complained of fever. Other common complaints included cough $(54 \%, 117$ of 216), vomiting (19\%, 40 of 216), headache (12\%, 26 of 216), and diarrhoea (12\%, 25 of 216). Prior to admission, 24\% (51 of 216) of patients had received antibiotics and 36\% (77 of 216) had received an anti-malarial drug including artemetherlumefantrine $(24 \%, 52$ of 216$)$ and quinine $(16 \%, 35$ of 216).

\section{Clinical and laboratory characteristics}

The majority of patients were febrile with a temperature $>38^{\circ} \mathrm{C}(71 \%, 153$ of 216$)$, tachycardic with a heart rate of $\geq 100$ beats/min (82\%, 177 of 216), and tachypneic with a respiratory rate $>20$ breaths/min (96\%, 208 of 216$)$. Half the patients $(50 \%, 106$ of 216$)$ had severe sepsis with evidence of end-organ dysfunction. Only 4\% (8 of 216) of patients had PCR proven malaria whereas bacteraemia was detected in 19\% (41 of 216) of patients and 11\% (24 of 216) had tuberculosis as defined by positive sputum LEDFM. The most common presumptive diagnosis at discharge was tuberculosis $(28 \%, 61$ of 216$)$ followed by pneumonia (26\%, 56 of 216; Table 3). Of those with positive blood cultures, Staphylococcus aureus was the most frequently identified pathogen $(66 \%, 27$ of 41$)$ followed by Salmonella species (20\%, 8 of 41) and streptococcal species (7\%, 3 of 41). Laboratory evaluation revealed leukocytosis (WBC count $>12,000 / \mathrm{ml}$ ) in $11 \%$ (24 of 215), anaemia (haemoglobin $<9 \mathrm{mg} / \mathrm{dL}$ ) in $43 \%$ (93 of 216) and thrombocytopenia (platelets $<150$ cells $/ \mu \mathrm{L}$ ) in $55 \%$ (118 of 215) of participants. Lactic acidosis (lactate $>4 \mathrm{mmol} / \mathrm{L}$ ) was identified in $42 \%$ (89 of 211 ) of patients.

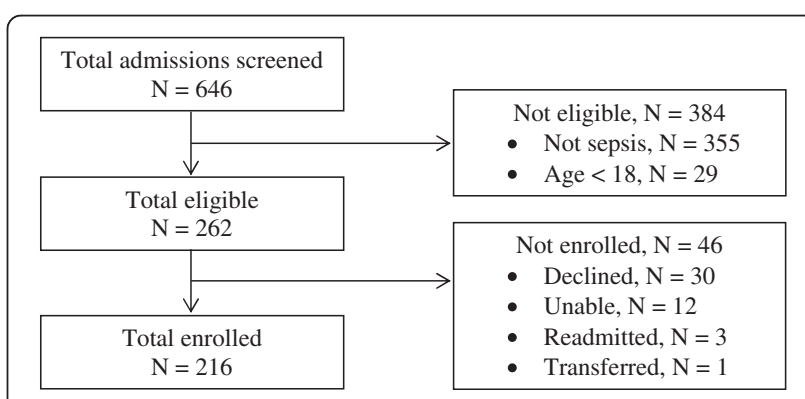

Figure 1 Flow diagram of patient enrolment for the study. 
Table 1 Summary statistics regarding demographics and clinical characteristics for a cohort of adult patients presenting with sepsis to a regional referral hospital in south-western Uganda $(n=216)$

\begin{tabular}{|c|c|}
\hline Characteristic & Summary statistic \\
\hline \multicolumn{2}{|l|}{ Demographics } \\
\hline Age (median, IQR) & $32(27-42)$ \\
\hline Female, n (\%) & $110(51)$ \\
\hline Married, n (\%) & $123(57)$ \\
\hline \multicolumn{2}{|l|}{ Educational attainment, n (\%) } \\
\hline None & $59(27)$ \\
\hline Primary & $121(56)$ \\
\hline Secondary & $32(15)$ \\
\hline Tertiary & $4(2)$ \\
\hline \multicolumn{2}{|l|}{ District of residence, $\mathrm{n}(\%)$} \\
\hline Mbarara & $72(33)$ \\
\hline Isingiro & $48(22)$ \\
\hline Kiruhura & $28(13)$ \\
\hline Other & $67(31)$ \\
\hline \multicolumn{2}{|l|}{ Clinical characteristics } \\
\hline \multicolumn{2}{|l|}{ Past medical history, n (\%) } \\
\hline None & $193(90)$ \\
\hline Diabetes mellitus & $6(3)$ \\
\hline Kaposi's Sarcoma & $6(3)$ \\
\hline Other & $10(5)$ \\
\hline \multicolumn{2}{|l|}{ Presenting symptoms, n (\%) } \\
\hline Fever & $194(90)$ \\
\hline Cough & $117(54)$ \\
\hline Vomiting & $40(19)$ \\
\hline Headache & $26(12)$ \\
\hline Diarrhoea & $25(12)$ \\
\hline Weight Loss & $17(8)$ \\
\hline Dyspnea & $15(7)$ \\
\hline Nightsweats & $13(6)$ \\
\hline \multicolumn{2}{|c|}{ Medications prior to admission, n (\%) } \\
\hline Antibiotics & $51(24)$ \\
\hline Artemether/lumafantrine & $52(24)$ \\
\hline Quinine & $35(16)$ \\
\hline \multicolumn{2}{|l|}{ Presenting signs, n (\%) } \\
\hline Fever $\left(>38^{\circ} \mathrm{C}\right)$ & $153(71)$ \\
\hline Tachycardia (100 beats/min) & $177(82)$ \\
\hline Tachypnea (>20 breaths/min) & $208(96)$ \\
\hline Hypotension (MAP < 65) & $62(29)$ \\
\hline Glasgow Coma Score <9 & $8(4)$ \\
\hline Severe Sepsis & $106(50)$ \\
\hline
\end{tabular}

Table 2 Summary statistics regarding test results and hospital treatments for a cohort of adult patients presenting with sepsis to a regional referral hospital in south-western Uganda $(n=216)$

\begin{tabular}{|c|c|}
\hline Characteristic & Summary statistic \\
\hline \multicolumn{2}{|l|}{ Test results, $\mathbf{n}$ (\% positive) } \\
\hline Anaemia (haemoglobin <9 mg/dl) & $93(43)$ \\
\hline Leukocytosis (WBC >12,000/ML) & $24(11)$ \\
\hline Thrombocytopenia (platelets $<150 / \mu \mathrm{L}$ ) & $118(55)$ \\
\hline Lactic Acidosis (lactate $>4 \mathrm{mmol} / \mathrm{L}$ ) & $89(42)$ \\
\hline Positive Blood Culture, n (\%) & $41(19)$ \\
\hline Staphylococcus aureus & $27(66)$ \\
\hline Salmonella spp. & $8(20)$ \\
\hline Streptococcus spp. & $3(7)$ \\
\hline Other/unidentified organism & $3(7)$ \\
\hline HIV seropositive with positive blood cultures, n (\%) & $19(9)$ \\
\hline Staphylococcus aureus & $17(89)$ \\
\hline Salmonella spp. & $1(5)$ \\
\hline Other/unidentified organism & $1(5)$ \\
\hline $\begin{array}{l}\text { HIV seronegative or serostatus unknown with } \\
\text { positive blood cultures, } \mathrm{n}(\%)\end{array}$ & $22(10)$ \\
\hline Staphylococcus aureus & $10(45)$ \\
\hline Salmonella spp. & $7(32)$ \\
\hline Streptococcus spp. & $3(14)$ \\
\hline Other/unidentified organism & $2(9)$ \\
\hline HIV seropositive, n (\%) & $122(56)$ \\
\hline CD4 count $<100$ cells/uL & $75(66)$ \\
\hline ART prior to admission & $44(36)$ \\
\hline Cotrimoxazole prior to admission & $80(66)$ \\
\hline Malaria test $\mathrm{n}$ (\% positive) & $9(4)$ \\
\hline Positive malaria smear & $2(1)$ \\
\hline Positive malaria rapid diagnostic test & $6(3)$ \\
\hline Positive malaria PCR & $8(4)$ \\
\hline Positive acid-fast bacteria sputum stain, n (\%) & $24(11)$ \\
\hline \multicolumn{2}{|l|}{ Hospital treatments received, $\mathbf{n}(\%)$} \\
\hline Artemether/lumafantrine & $33(15)$ \\
\hline Quinine & $45(21)$ \\
\hline Antibiotics & $187(87)$ \\
\hline Anti-tuberculosis medicines & $16(7)$ \\
\hline
\end{tabular}

\section{Malaria diagnosis}

Malaria was identified by PCR in eight patients. There were six cases of $P$. falciparum and two cases of $P$. vivax infection. RDT and blood slide identified five and two of these eight patients respectively. A positive RDT result was $62.5 \%$ (95\% CI 24.5-91.5) sensitive and 99.5\% (95\% CI 97.3-100) specific for PCR proven malaria with an $83.3 \%$ positive predictive value and $98.6 \%$ negative predictive value. The sensitivity and specificity of a positive 
Table 3 Discharge diagnoses and outcomes among patients presenting with sepsis to a regional referral hospital in south-western Uganda

\begin{tabular}{llll}
\hline & Diagnosis at discharge, $\mathbf{n}(\%)$ & Length of stay Median (IQR) & Mortality rate \% \\
\hline $\begin{array}{l}\text { Total cohort } \\
\text { Definitive diagnosis }\end{array}$ & $216(100)$ & $5(3-8)$ & 20.0 \\
Malaria & $8(3)$ & $4(3-4.5)$ & 12.5 \\
Bacteraemia & $41(19)$ & $5.5(3-8.5)$ & 26.8 \\
Pulmonary tuberculosis & $24(11)$ & $5.5(2.5-13.5)$ & 25.0 \\
Presumptive diagnosis & & & 11.1 \\
Malaria & $18(8)$ & $3(2-5)$ & 24.6 \\
Tuberculosis & $61(28)$ & $7(4-11)$ & 10.5 \\
Enteric fever & $19(9)$ & $4(4-10)$ & 12.5 \\
Community acquired pneumonia & $56(26)$ & $4(3-6)$ & $6(3-12)$ \\
Fever of unknown origin & $17(8)$ & $5(3-9)$ & 23.5 \\
Other diagnosis, or unspecified & $65(30)$ & & 21.5 \\
\hline
\end{tabular}

blood slide for a PCR proven malaria diagnosis was $25 \%$ (95\% CI 3.2-65.1) and 100\% (95\% CI 98.2-100) respectively with a positive predictive value of $100 \%$ and negative predictive value of $97.2 \%$. A clinical discharge diagnosis of malaria was 62\% (95\% CI 24.5-91.5) sensitive and 99.5\% (95\% CI 97.3-100) specific for PCR proven malaria.

Due to the low prevalence of malaria in the cohort, clinical correlates associated with malaria were difficult to determine; however, cough was less common in those with malaria $(13 \%, 1$ of 8 vs 56\%, 116 of 208 in patients without malaria, $\mathrm{p}=0.02)$. A final clinical discharge diagnosis of malaria was given to $50 \%$ (4 of 8 ) of patients with malaria compared to $7 \%$ (14 of 208) in those without malaria $(\mathrm{p}<0.01)$. Anti-malarial treatment was provided to $63 \%$ (5 of 8 ) of patients with malaria and $23 \%$ (48 of 208) of those without malaria $(p=0.01)$. Antibacterial therapy was provided to $63 \%$ (five of eight) and $88 \%$ (182 of 208) of patients with and without malaria respectively $(\mathrm{p}=0.05)$.

\section{Outcomes}

The median length of stay was five days (IQR 3-8, range 0-41 days) and in-hospital death occurred in 19\% (42 of 216) of the cohort. Among those with a definitive diagnosis of sepsis, mortality was $26.8 \%$ in those with bacteraemia, $25 \%$ for those with LED-FM sputum positive tuberculosis, and $12.5 \%$ in those with malaria. Mortality rates for those with presumptive diagnoses were $24.6 \%$ for tuberculosis, $23.5 \%$ for fever of unknown origin, $21.5 \%$ for an unknown diagnosis, $12.5 \%$ for community acquired pneumonia, $11.1 \%$ for malaria, and $10.5 \%$ for enteric fever (Table 3). In the univariable analysis, GCS $<9$, severe sepsis, and lactate $>4 \mathrm{mmol} / \mathrm{L}$ were all significantly associated with in-hospital mortality while fever was associated with survival ( $p<0.05$; Table 4 ). In the multivariable regression model, the adjusted relative risk (RR) for in-hospital mortality associated with a GCS $<9$ was 4.8 with a $95 \%$ confidence interval (CI) of 1.8 12.8 and $\mathrm{p}<0.01$. Severe sepsis was also independently associated with in-hospital mortality (RR 2.1, 95\% CI 1.0-4.1, $\mathrm{p}=0.04)$. Lactate was removed from the multivariable model due to collinearity with severe sepsis. In contradistinction, fever was independently associated with reduced in-hospital mortality (RR 0.5, 95\% CI 0.2-0.9, $\mathrm{p}=0.02$ ).

\section{Discussion}

In adult patients presenting to a regional referral hospital in south-western Uganda where malaria transmission is moderate, malaria was an uncommon cause of sepsis. These findings have implications for empirical treatment of sepsis in this region and emphasize the importance of the determination of microbiological diagnoses when treating sepsis. Based on these data, malaria as a sole cause of sepsis should be considered a diagnosis of exclusion among patients presenting with sepsis in similar settings. Accordingly, since the negative predictive value of RDT is so high treatment for malaria should be limited to those in whom malaria diagnosis has been made through a positive RDT.

The low malaria prevalence of approximately 4\% in this cohort of adult patients with sepsis contrasts with national Ugandan malaria epidemiology. As a whole, Uganda has a high malaria prevalence (30-50\%) which may be increasing [14]. Additionally, annual entomological inoculation rates (EIRs) vary in Uganda from four infective bites per person year in the south-western highlands to > 1,500 infective bites per person per year in Apac in the central part of the country [15]. Malaria admissions increased in children in Uganda from 1999 until 2009 but similar data are not available for adults [15]. In contrast to the rest of the country, Mbarara District has had a 
Table 4 Incidence of mortality in adult patients presenting to the medical ward at Mbarara Regional Referral Hospital with sepsis (Multivariable regression model adjusted for predictors of mortality that met inclusion by a p-value $<0.25$ in the univariable model)

\begin{tabular}{|c|c|c|c|c|c|c|c|}
\hline Characteristic & $\begin{array}{l}\text { Incidence of } \\
\text { mortality }\end{array}$ & $\begin{array}{l}\text { Incidence } \\
\text { rate ratio }\end{array}$ & $95 \% \mathrm{Cl}$ & p-value & $\begin{array}{l}\text { Adjusted incidence } \\
\text { rate ratio }\end{array}$ & $95 \% \mathrm{Cl}$ & $p$-value \\
\hline Entire cohort & 19.4 & - & - & - & & & \\
\hline Age (each 10 years) & - & 1.02 & $0.84-1.27$ & 0.78 & - & & \\
\hline Male (REF) & 24.5 & & & & & & \\
\hline Female & 14.6 & 0.59 & $0.32-1.11$ & $0.10^{*}$ & 0.57 & $0.29-1.10$ & 0.10 \\
\hline Single (REF) & 22.0 & & & & - & & \\
\hline Married & 17.9 & 0.81 & $0.44-1.49$ & 0.51 & & & \\
\hline \multicolumn{8}{|l|}{ Educational attainment } \\
\hline Less than primary (REF) & 17.8 & & & & & & \\
\hline Greater than primary & 27.8 & 1.56 & $0.77-3.18$ & $0.22^{*}$ & 1.45 & $0.70-3.03$ & 0.32 \\
\hline \multicolumn{8}{|l|}{ Clinical characteristics } \\
\hline No antibiotics prior (REF) & 18.8 & & & & - & & \\
\hline Antibiotics prior & 21.6 & 1.15 & $0.58-2.28$ & 0.69 & & & \\
\hline No fever (REF) & 31.8 & & & & & & \\
\hline Fever & 14.4 & 0.45 & $0.25-0.83$ & $0.01^{*}$ & 0.46 & $0.24-0.88$ & 0.02 \\
\hline No tachycardia (REF) & 23.1 & & & & - & & \\
\hline Tachycardia & 18.6 & 0.81 & $0.39-1.69$ & 0.57 & & & \\
\hline No tachypnea (REF) & 12.5 & & & & - & & \\
\hline Tachypnea & 19.7 & 1.57 & $0.22-11.46$ & 0.65 & & & \\
\hline No hypotension (REF) & 19.5 & & & & - & & \\
\hline Hypotension & 19.4 & 0.99 & $0.51-1.94$ & 0.99 & & & \\
\hline GCS >9 (REF) & 17.1 & & & & & & \\
\hline Glasgow Coma Score <9 & 75.0 & 4.39 & $1.85-10.44$ & $<0.01^{*}$ & 4.81 & $1.80-12.8$ & $<0.01$ \\
\hline Sepsis (REF) & 11.2 & & & & & & \\
\hline Severe sepsis & 27.4 & 2.44 & $1.24-4.78$ & $0.01^{*}$ & 2.07 & $1.03-4.14$ & 0.04 \\
\hline No aenemia (REF) & 21.3 & & & & - & & \\
\hline Anaemia & 17.2 & 0.81 & $0.43-1.50$ & 0.50 & & & \\
\hline No leuckocytosis (REF) & 18.3 & & & & - & & \\
\hline Leukocytosis & 29.2 & 1.59 & $0.71-3.58$ & 0.26 & & & \\
\hline No thrombocytopaenia (REF) & 17.5 & & & & - & & \\
\hline Thrombocytopaenia & 21.2 & 1.21 & $0.65-2.24$ & 0.55 & & & \\
\hline No lactic acidosis (REF) & 13.1 & & & & - & & \\
\hline Lactic acidosis & 28.1 & 2.14 & $1.14-4.01$ & $0.02^{*}$ & & & \\
\hline HIV seronegative (REF) & 19.2 & & & & - & & \\
\hline HIV seropositive & 19.7 & 1.03 & $0.56-1.89$ & 0.93 & & & \\
\hline \multicolumn{8}{|l|}{ Definitive diagnosis at discharge } \\
\hline Negative blood culture (REF) & 17.9 & & & & & & \\
\hline Positive blood culture & 26.8 & 1.50 & $0.75-2.98$ & $0.25^{*}$ & 1.40 & $0.68-2.87$ & 0.36 \\
\hline Negative or no sputum smear for AFB (REF) & 18.8 & & & & - & & \\
\hline Positive AFB sputum smear & 25.0 & 1.33 & $0.56-3.16$ & 0.51 & & & \\
\hline No confirmed malaria (REF) & 19.1 & & & & - & & \\
\hline Confirmed malaria & 33.3 & 1.75 & $0.43-7.24$ & 0.44 & & & \\
\hline
\end{tabular}


Table 4 Incidence of mortality in adult patients presenting to the medical ward at Mbarara Regional Referral Hospital with sepsis (Multivariable regression model adjusted for predictors of mortality that met inclusion by a $\mathrm{p}$-value $<0.25$ in the univariable model) (Continued)

\begin{tabular}{|c|c|c|c|c|c|}
\hline \multicolumn{6}{|l|}{ Clinical diagnosis at discharge } \\
\hline Not malaria (REF) & 20.2 & & & & - \\
\hline Malaria & 11.1 & 0.55 & $0.13-2.28$ & 0.41 & \\
\hline Not tuberculosis (REF) & 17.4 & & & & - \\
\hline Tuberculosis & 24.6 & 1.41 & $0.75-2.65$ & 0.28 & \\
\hline Not enteric fever REF) & 20.3 & & & & - \\
\hline Enteric fever & 10.5 & 0.52 & $0.12-2.15$ & 0.36 & \\
\hline Not pneumonia (REF) & 21.9 & & & & - \\
\hline Pneumonia & 12.5 & 0.57 & $0.24-1.29$ & 0.18 & \\
\hline Not fever of unknown origin (REF) & 19.1 & & & & - \\
\hline Fever of unknown origin & 23.5 & 1.23 & $0.44-3.45$ & 0.69 & \\
\hline No other diagnosis (REF) & 18.5 & & & & - \\
\hline Other diagnosis, or unspecified & 21.5 & 1.16 & $0.61-2.21$ & 0.65 & \\
\hline
\end{tabular}

heterogeneous decrease in malaria in children [11]. Depending on the area within the district and the amount of rainfall, Mbarara has an EIR of 11-100/year and a prevalence of $4-23 \%[11,14]$.

The reason for the low malaria prevalence in this cohort compared to the general population may be due to several factors. First, epidemiological data are generally derived from children as adults have improved immunological control of malaria and therefore lower prevalence of infection even in the setting of HIV infection [2]. Severe malaria may occur more often in adults who have waxing and waning immunity to malaria where malaria transmission is unstable and prone to epidemics, such as the highlands of south-western Uganda which does not include Mbarara District [16]. Second, many of the patients $(40 \%)$ had already received anti-malarial therapy prior to admission. Therefore, any incidental parasitaemia that was present in these patients was likely eradicated prior to admission.

The RDT used in this study employed HRP-II to identify Plasmodium falciparum and $\mathrm{pLDH}$ to identify non-falciparum species. HRP-II antigen tests are very sensitive and positive results can persist 14 days or more after treatment whereas $\mathrm{pLDH}$ provides a negative RDT result within 3 days of treatment $[17,18]$. There were only 6 positive RDT results in this study. If malaria was a significant cause of sepsis then more positive RDT results would be expected despite any preceding anti-malarial therapy. It cannot be completely ruled out that some of the patients already on treatment could have been falselynegative for malaria by RDT, but these cases should have been captured by the more sensitive PCR test. Although microscopy and the degree of parasitaemia are more correlated with clinical symptoms, PCR was chosen as the gold-standard in this study in order to apply the most sensitive method to detect malaria parasitaemia. The use of PCR allowed capture of the rare patient that had malaria but had pre-hospital anti-malarial treatment and a subsequently negative malaria slide or RDT result.

A diagnosis of malaria was not associated with increased mortality in this study or in similar studies. Moreover, in contrast to receipt of early antibacterial therapy, receipt of anti-malarial drugs did not improve mortality outcomes in this study or a study of fluid resuscitation in severe sepsis performed in Uganda [19]. In a study performed in a high malaria prevalence area of Tanzania, malaria was not a frequent cause of severe febrile illness in adults [20]. That study used RDTs and blood smears but not PCR to define the presence of malaria parasitaemia. Taken together, the results of this study suggest that parasitaemia occurs very rarely in this patient population and that malaria is infrequently a cause of adult sepsis.

This and prior studies of severe sepsis in Uganda have revealed non-Typhi Salmonella, Streptococcus pneumoniae, and Staphylococcus aureus as common pathogens [5,19,21]. A close temporal association between bacteraemia and malaria has also recently been made clear [22]. There were no cases of dual infection in the current study. However, pre-hospitalization use of antibacterial drugs may have obscured the ability to detect bacteria in blood cultures for some patients in this study. Tuberculosis was a leading discharge diagnosis for this cohort and was associated with approximately $25 \%$ mortality. Other studies in SSA have documented Mycobacterium tuberculosis (MTb) as a common cause of bloodstream infection in hospitalized patients and these patients have high mortality with $50 \%$ of patients dying within 36 days 
in one study $[19,21,23]$. Despite these findings, it is rare that antituberculous drugs are included in empiric sepsis therapy in MTb endemic regions such as Uganda. Further investigation into MTb rapid diagnostics and empirical anti-MTb therapy in the setting of sepsis in this region are needed [24]. In this study, patients with altered mental status and severe sepsis were at highest risk of death. This is consistent with prior work from the same institution and others in the region $[5,19,21]$.

There are several limitations of this study. Patients were not evaluated through an entire calendar year and times of high malaria transmission may have been missed. The enrolment period for this study did include one of the rainiest times of year in Mbarara (MarchMay) which accordingly is a time of high community malaria prevalence [11]. Therefore, it is unlikely that malaria prevalence in adult patients admitted with sepsis would be higher at any other time. However, enrolment of adult septic patients throughout a calendar year might provide additional information on temporal changes in malaria prevalence. Due to the small number of patients with PCR proven malaria in this study, it is not possible to draw definitive conclusions regarding the relative abilities of blood slides and RDTs to diagnose malaria in adult septic patients. These data do suggest that a positive finding by either modality is specific for malaria. Beyond malaria diagnostics, the ability to determine the aetiology of sepsis in these patients was limited. Given the high prevalence of mycobacteraemia that has recently been described in similar patients in SSA it is likely that many of these patients were infected with MTb $[19,23]$. This conjecture is supported by the large proportion of patients that were assigned a presumptive (28\%) or definitive (11\%) discharge diagnosis of tuberculosis. Additionally, it was not possible to detect rickettsial diseases, Q-fever, leptospirosis, brucellosis, or histoplasmosis, which have recently been increasingly recognized in the region [25-28].

Despite these limitations, data from this study lend strong support to the notion that malaria is an uncommon cause of sepsis in adult inpatients in south-western Uganda. Based on the findings of this study, the following recommendations are made to practitioners providing care to patients with sepsis in similar settings. First, thorough diagnostic evaluations should be pursued where possible to evaluate for non-malarial causes of sepsis. Second, as recommended by the World Health Organization, anti-malarial therapy should be reserved for patients with a positive RDT or blood slide result to minimize over-prescribing and development of resistance. A bacterial cause of sepsis was identified in $\sim 20 \%$ of these patients and it is suggested that empirical treatment of sepsis in this region should include coverage of staphylococci, streptococci, and Salmonella species. Unfortunately, the vast majority of patients did not have a confirmed microbiological diagnosis for their sepsis. Further studies are needed to discern the role for empirical anti-MTb therapy in patients presenting with sepsis in high HIV prevalent MTb endemic areas. Improved diagnostic capacity, including development of point-of-care testing, for use in regional referral hospitals in resource-limited settings may improve targeted and empirical therapy for sepsis, reduce development of resistance to anti-malarial agents, and improve outcomes. Finally, given the high prevalence of HIV in this region all patients admitted with sepsis should be tested for HIV according to national guidelines.

\section{Conclusions}

In Mbarara which is located south-western Uganda and where community malaria prevalence is $4-23 \%$ malaria was an infrequent cause of sepsis in adults. The ability to detect parasitaemia was maximized through use of PCR technology. Using this sensitive laboratory method, only 8 out of 216 patients were diagnosed with malaria. In contrast, bacteraemia was found in 41 (19\%) patients. Additionally, tuberculosis was a definitive diagnosis for $24(11 \%)$ and presumptive diagnosis for 61 (28\%) of these patients. When treating adult septic patients in similar regions a thorough evaluation of non-malaria diagnoses with particular emphasis on identifying bacteraemia and tuberculosis is recommended.

\section{Abbreviations}

SSA: Sub-Saharan Africa; HIV: Human immunodeficiency virus; RDT: Rapid diagnostic test; PCR: Polymerase chain reaction; MRRH: Mbarara Regional Referral Hospital; MUST: Mbarara University of Science and Technology; WBC: White blood cell concentration; GCS: Glasgow coma scale; HRP-II: Histidinerich protein II; pLDH: Plasmodium lactate dehydrogenase; LED-FM: Light-emitting diode fluorescent microscopy; DNA: Deoxyribonucleic acid;

RNA: Ribonucleic acid; CD: Cluster of differentiation; IQR: Interquartile range; RR: Relative risk; Cl: Confidence interval; EIR: Entomological inoculation rate; MTb: Mycobacterium tuberculosis.

\section{Competing interests}

The authors declare that they have no competing interests.

\section{Authors' contributions}

MAA helped design the study, enrolled patients, analysed the data, and helped to draft the manuscript. MJS analysed the data and helped to draft the manuscript. DN, AN, MN, and JM-A performed laboratory analyses including malaria PCR and bacterial cultures and helped to draft the manuscript. RM and LAW helped to design the study, analyse the data, and draft the manuscript. YB helped to design the study, supervised the malaria PCR work, helped to analyse the data, and helped to draft the manuscript. CCM conceived of and designed the study, coordinated the study, helped to analyse the data, and drafted the manuscript. All authors read and approved the final manuscript.

\section{Acknowledgements}

Funding for the study was provided to MAA and CCM by the Pfizer Initiative in International Health and the Center for Global Health at the University of Virginia. This initiative was conceived to fund global infectious disease research and exchange programmes between post-doctoral fellows and students from the University of Virginia and several international partners to conduct research on global health issues. The major purpose of this 
programme is to foster and enhance bidirectional research training. An independent board at the University of Virginia determines the research proposals that are funded. Pfizer, Inc provided funds to promote the initiative but has no role in the planning or execution of research protocols, including the study described in this article. MAA also received salary, tuition, and research support from the Lacor Hospital administration, Gulu, Uganda. MS received salary and research support from the Harvard Institute for Global Health, the Fogarty International Clinical Research Scholars and Fellows Program at Vanderbilt University (R24 TW007988), and the National Institutes of Health (T32 Al007433)

\section{Author details}

${ }^{1}$ Faculty of Medicine, Mbarara University of Science and Technology, Mbarara, Uganda. ${ }^{2}$ Division of Infectious Diseases, Massachusetts General Hospital, Harvard Medical School, Boston, MA, USA. ${ }^{3}$ Epicentre Mbarara Research Base, Mbarara, Uganda. ${ }^{4}$ Department of Microbiology, Mbarara University of Science and Technology, Mbarara, Uganda. ${ }^{5}$ Department of Medicine, Division of Infectious Diseases and International Health, University of Virginia, Charlottesville, VA, USA.

\section{Received: 28 February 2013 Accepted: 29 April 2013}

Published: 1 May 2013

\section{References}

1. Lozano R, Naghavi M, Foreman K, Lim S, Shibuya K, Aboyans V, Abraham J, Adair T, Aggarwal R, Ahn SY, Alvarado M, Anderson HR, Anderson LM, Andrews KG, Atkinson C, Baddour LM, Barker-Collo S, Bartels DH, Bell ML, Benjamin EJ, Bennett D, Bhalla K, Bikbov B, Bin AA, Birbeck G, Blyth F, Bolliger I, Boufous S, Bucello C, Burch M, et al: Global and regional mortality from 235 causes of death for 20 age groups in 1990 and 2010: a systematic analysis for the Global Burden of Disease Study 2010. Lancet 2013, 380:2095-2128.

2. Francesconi $P$, Fabiani M, Dente MG, Lukwiya M, Okwey R, Ouma J, Ochakachon R, Cian F, Declich S: HIV, malaria parasites, and acute febrile episodes in Ugandan adults: a case-control study. AIDS 2001, 15:2445-2450

3. French N, Nakiyingi J, Lugada E, Watera C, Whitworth JA, Gilks CF: Increasing rates of malarial fever with deteriorating immune status in HIV-1-infected Ugandan adults. AIDS 2001, 15:899-906.

4. Pullan RL, Bukirwa H, Staedke SG, Snow RW, Brooker S: Plasmodium infection and its risk factors in eastern Uganda. Malar J 2010, 9:2.

5. Ssekitoleko R, Pinkerton R, Muhindo R, Bhagani S, Moore CC: Aggregate evaluable organ dysfunction predicts in-hospital mortality from sepsis in Uganda. Am J Trop Med Hyg 2011, 85:697-702

6. Whitworth J, Morgan D, Quigley M, Smith A, Mayanja B, Eotu H, Omoding N, Okongo M, Malamba S, Ojwiya A: Effect of HIV-1 and increasing immunosuppression on malaria parasitaemia and clinical episodes in adults in rural Uganda: a cohort study. Lancet 2000, 356:1051-1056.

7. Murray CJ, Rosenfeld LC, Lim SS, Andrews KG, Foreman KJ, Haring D, Fullman N, Naghavi M, Lozano R, Lopez AD: Global malaria mortality between 1980 and 2010: a systematic analysis. Lancet 2012, 379:413-431.

8. Nankabirwa J, Zurovac D, Njogu JN, Rwakimari JB, Counihan H, Snow RW, Tibenderana JK: Malaria misdiagnosis in Uganda-implications for policy change. Malar J 2009, 8:66.

9. Reyburn H, Mbatia R, Drakeley C, Carneiro I, Mwakasungula E, Mwerinde O, Saganda K, Shao J, Kitua A, Olomi R, Greenwood BM, Whitty CJ: Overdiagnosis of malaria in patients with severe febrile illness in Tanzania: a prospective study. BMJ 2004, 329:1212.

10. Kumar A, Roberts D, Wood KE, Light B, Parrillo JE, Sharma S, Suppes R, Feinstein D, Zanotti S, Taiberg L, Gurka D, Kumar A, Cheang M: Duration of hypotension before initiation of effective antimicrobial therapy is the critical determinant of survival in human septic shock. Crit Care Med 2006, 34:1589-1596.

11. De Beaudrap P, Nabasumba C, Grandesso F, Turyakira E, Schramm B, Boum Y, Etard JF: Heterogeneous decrease in malaria prevalence in children over a six-year period in south-western Uganda. Malar J 2011, 10:132.

12. Moore CC, Jacob ST, Pinkerton R, Banura P, Meya DB, Reynolds SJ, Kenya-Mugisha N, Mayanja-Kizza H, Scheld WM: Treatment of severe sepsis with artemether-lumefantrine is associated with decreased mortality in Ugandan patients without malaria. Am J Trop Med Hyg 2009, 80:723-728.

13. Snounou G, Viriyakosol S, Zhu XP, Jarra W, Pinheiro L, do Rosario VE, Thaithong S, Brown KN: High sensitivity of detection of human malaria parasites by the use of nested polymerase chain reaction. Mol Biochem Parasitol 1993, 61:315-320.

14. Yeka A, Gasasira A, Mpimbaza A, Achan J, Nankabirwa J, Nsobya S, Staedke SG, Donnelly MJ, Wabwire-Mangen F, Talisuna A, Dorsey G, Kamya MR, Rosenthal PJ: Malaria in Uganda: challenges to control on the long road to elimination: I. Epidemiology and current control efforts. Acta Trop 2012, 121:184-195.

15. Okiro EA, Bitira D, Mbabazi G, Mpimbaza A, Alegana VA, Talisuna AO, Snow RW: Increasing malaria hospital admissions in Uganda between 1999 and 2009. BMC Med 2011, 9:37.

16. Idro R, Bitarakwate E, Tumwesigire S, John CC: Clinical manifestations of severe malaria in the highlands of southwestern Uganda. Am J Trop Med Hyg 2005, 72:561-567.

17. Fogg C, Twesigye R, Batwala V, Piola P, Nabasumba C, Kiguli J, Mutebi F, Hook C, Guillerm M, Moody A, Guthmann JP: Assessment of three new parasite lactate dehydrogenase (pan-pLDH) tests for diagnosis of uncomplicated malaria. Trans R Soc Trop Med Hyg 2008, 102:25-31.

18. Swarthout TD, Counihan H, Senga RK, van den Broek I: Paracheck-Pf accuracy and recently treated Plasmodium falciparum infections: is there a risk of over-diagnosis? Malar J 2007, 6:58.

19. Jacob ST, Banura P, Baeten JM, Moore CC, Meya D, Nakiyingi L, Burke R, Horton CL, lga B, Wald A, Reynolds SJ, Mayanja-Kizza H, Scheld WM: The impact of early monitored management on survival in hospitalized adult Ugandan patients with severe sepsis: a prospective intervention study*. Crit Care Med 2012, 40:2050-2058.

20. Nadjm B, Mtove G, Amos B, Walker NF, Diefendal H, Reyburn H, Whitty CJ: Severe febrile illness in adult hospital admissions in Tanzania: a prospective study in an area of high malaria transmission. Trans $R$ Soc Trop Med Hyg 2012, 106:688-695.

21. Jacob ST, Moore CC, Banura P, Pinkerton R, Meya D, Opendi P, Reynolds SJ, Kenya-Mugisha N, Mayanja-Kizza H, Scheld WM: Severe sepsis in two Ugandan hospitals: a prospective observational study of management and outcomes in a predominantly HIV-1 infected population. PLOS One 2009, 4:e7782.

22. Scott JA, Berkley JA, Mwangi I, Ochola L, Uyoga S, Macharia A, Ndila C, Lowe BS, Mwarumba S, Bauni E, Marsh K, Williams TN: Relation between falciparum malaria and bacteraemia in Kenyan children: a populationbased, case-control study and a longitudinal study. Lancet 2011, 378:1316-1323.

23. Crump JA, Ramadhani HO, Morrissey AB, Saganda W, Mwako MS, Yang LY, Chow SC, Njau BN, Mushi GS, Maro VP, Reller LB, Bartlett JA: Bacteremic disseminated tuberculosis in sub-saharan Africa: a prospective cohort study. Clin Infect Dis 2012, 55:242-250.

24. Wallis RS, Pai M, Menzies D, Doherty TM, Walzl G, Perkins MD, Zumla A Biomarkers and diagnostics for tuberculosis: progress, needs, and translation into practice. Lancet 2010, 375:1920-1937.

25. Biggs HM, Bui DM, Galloway RL, Stoddard RA, Shadomy SV, Morrissey AB, Bartlett JA, Onyango JJ, Maro VP, Kinabo GD, Saganda W, Crump JA: Leptospirosis among hospitalized febrile patients in northern Tanzania. Am J Trop Med Hyg 2011, 85:275-281.

26. Bouley AJ, Biggs HM, Stoddard RA, Morrissey AB, Bartlett JA, Afwamba IA, Maro VP, Kinabo GD, Saganda W, Cleaveland S, Crump JA: Brucellosis among hospitalized febrile patients in Northern Tanzania. Am J Trop Med Hyg 2012, 87:1105-1111.

27. Lofgren SM, Kirsch EJ, Maro VP, Morrissey AB, Msuya LJ, Kinabo GD, Saganda W, Diefenthal HC, Ramadhani HO, Wheat L, Crump JA: Histoplasmosis among hospitalized febrile patients in northern Tanzania. Trans R Soc Trop Med Hyg 2012, 106:504-507.

28. Prabhu M, Nicholson WL, Roche AJ, Kersh GJ, Fitzpatrick KA, Oliver LD, Massung RF, Morrissey AB, Bartlett JA, Onyango JJ, Maro VP, Kinabo GD, Saganda W, Crump JA: Q fever, spotted fever group, and typhus group rickettsioses among hospitalized febrile patients in northern Tanzania. Clin Infect Dis 2011, 53:e8-e15.

doi:10.1186/1475-2875-12-146

Cite this article as: Auma et al:: Malaria is an uncommon cause of adult sepsis in south-western Uganda. Malaria Journal 2013 12:146. 